\title{
BOUNDED HARMONIC FUNCTIONS ON COVERINGS
}

\author{
DOMINGO TOLEDO*
}

(Communicated by David J. Ebin)

\begin{abstract}
We prove that the space of bounded harmonic functions on a Galois cover of a compact Riemannian manifold is either one dimensional or infinite dimensional.
\end{abstract}

The purpose of this note is to prove the following

THEOREM. Let $M$ be a Riemannian manifold which is a Galois covering of a compact Riemannian manifold. Then the space of bounded harmonic functions on $M$ is either one dimensional or infinite dimensional.

Since the space of bounded harmonic functions always contains the one dimensional space of constant functions, we could restate the theorem as saying that the space of bounded harmonic functions on a Galois covering, if nontrivial, must be of infinite dimension. The proof is based on two theorems of Lyons and Sullivan [2]. We refer to this paper for motivation.

I am grateful to Peter $\mathrm{Li}$ for bringing this problem to my attention and several helpful discussions, and to Steve Gersten for a reference used for Lemma 2.

PROOF. Let $V$ denote the space of bounded harmonic functions on $M$, with the sup norm, and let $\Gamma$ denote the covering group. $\Gamma$ acts on $V$ by isometries, thus we get a representation $\rho: \Gamma \rightarrow \operatorname{Isom}(V)$. Observe that we may assume that $\rho$ is injective, for if $K$ denotes the kernel of $\rho$, the space of bounded harmonic functions on $M / K$ is isomorphic to $V$, and $M / K$ is a Galois covering of $M / \Gamma$.

LEMMA 1. If $V$ is finite dimensional, then $\Gamma$ is an amenable group.

ProOF. Let $C \subset V$ denote the cone of positive functions, and let $G \subset \operatorname{Isom}(V)$ be the subgroup of isometries of $V$ that preserve $C$ and act as the identity on the subspace of constant functions. The image of $\rho$ lies in $G$. Let $l$ be a linear functional on $V$ which is positive on $C$ and has value one on the constant function one. If $V$ is finite dimensional $G$ is a compact group, so $l$ can be averaged over $G$ to a $G$-invariant map (hence $\Gamma$-invariant) linear functional $\lambda$ with the same properties. The proof of Theorem $3^{\prime}$ of $[2, \S 5]$ gives a $\Gamma$-equivariant map $\psi: l^{\infty}(\Gamma) \rightarrow V$ which takes the positive cone in $l^{\infty}(\Gamma)$ to $C$ and the constant function one to the constant function one. The composition $\lambda \psi$ is then an invariant mean on $\Gamma$, thus proving the lemma.

Received by the editors November 4, 1986.

1980 Mathematics Subject Classification (1985 Revision). Primary 53C20.

* Supported by NSF Grant DMS8503765. 
Next we observe that if $V$ is finite dimensional the compact group $G$ leaves an inner product invariant, so $\Gamma$ has a faithful representation in an orthogonal group $O(n)$.

LEMMA 2. Let $\Gamma$ be a finitely generated amenable subgroup of $O(n)$. Then $\Gamma$ contains an abelian subgroup of finite index.

PROOF. Since an amenable group cannot contain a nonabelian free group, by a well-known theorem of Tits [3] $\Gamma$ contains a solvable subgroup $\Gamma^{\prime}$ of finite index. $\Gamma^{\prime}$ then has a subgroup $\Gamma^{\prime \prime}$ of finite index that can be put, over the complex numbers, in upper triangular form [1, Corollary, p. 243]. Since $\Gamma^{\prime \prime} \subset O(n)$, the real vector space on which it acts splits as a direct sum of two and one-dimensional real subspaces which are $\Gamma^{\prime \prime}$-invariant. Thus we get an injective homomorphism $\Gamma^{\prime \prime} \rightarrow H$, where $H$ is a product of groups isomorphic to $O(2)$ or $O(1) . H$ has an abelian subgroup of finite index, and its intersection with $\Gamma^{\prime \prime}$ is the desired abelian subgroup of finite index in $\Gamma$.

We can now complete the proof of the theorem. If $V$ is finite dimensional then $\Gamma$ contains an abelian subgroup $\Gamma^{\prime}$ of finite index. $M$ is then a Galois covering of the compact manifold $M / \Gamma^{\prime}$, and by Theorem 1 of [2], $M$ has no nonconstant positive harmonic function. Thus $\operatorname{dim} V=1$, and the theorem follows.

REMARK. The proof of Lemma 1 shows that the conclusion of Theorem 3 of [2] can be strengthened to "the space of bounded harmonic functions is infinite dimensional."

ADDED IN PROOF. Similar results have been obtained by Y. Kifer, "Bounded harmonic functions on non-amenable covers of compact manifolds", Israel Jour. Math. 61 (1988), 170-178.

\section{REFERENCES}

1. A. Borel, Linear algebraic groups, Benjamin, New York, 1969.

2. T. Lyons and D. Sullivan, Function Theory, Random paths and covering spaces, J. Differential Geom. 19 (1984), 299-323.

3. J. Tits, Free subgroups in linear groups, J. of Algebra 20 (1972), 250-270.

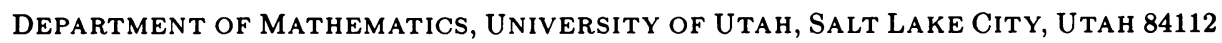

